\title{
Coherent Terahertz Radiation Generation Assisted by Low-Temperature Optical Phonon Emission: Achievements and Perspectives
}

\author{
P. Shiktorov ${ }^{a}$, E. Starikov ${ }^{a}$, V. GruŽinskis ${ }^{a}$, L. VARANi $^{b}$ \\ AND L. REGGIANI ${ }^{c}$ \\ ${ }^{a}$ Semiconductor Physics Institute, Goštauto 11, LT-01108, Vilnius, Lithuania \\ ${ }^{b}$ Institut d'Électronique du Sud (CNRS UMR 5214) \\ Université Montpellier 2
}

Pl. Eugène Bataillon, 34095 Montpellier Cedex 5, France

${ }^{c}$ Dipartimento di Ingegneria dell'Innovazione, Università del Salento and CNISM

Via Arnesano s/n, I-73100 Lecce, Italy

\begin{abstract}
The conditions for $\mathrm{THz}$ radiation generation caused by electron transite time resonance in momentum and real spaces under low-temperature opticalphonon emission are analyzed. It is shown that such a phenomenon provides a unique possibility to realize the sub- $\mathrm{THz}$ and $\mathrm{THz}$ radiation generation at the border of the electrooptical and electronic techniques by using both the approaches: (i) amplification of transverse electromagnetic waves in $3 \mathrm{D}$ bulk materials and 2D quantum wells, and (ii) longitudinal current instabilities in submicron and overmicron $n^{+} n n^{+}$diodes.
\end{abstract}

PACS numbers: 72.20.Ht, 72.30.+q, 73.63.Hs

\section{Introduction}

It is well known that the $\mathrm{THz}$ represents a border between frequency regions exploiting two different ways to achieve electromagnetic generation, namely: from the bottom ultrahigh frequency electronics and from the top far-infrared photonics. In the former case, the generation is obtained due to longitudinal current instabilities (as in the Gunn devices, impact ionization avalanche transit-time (IMPATT) diodes, etc.) which exploit negative differential conductivity of N- or S-type (static NDC). In the latter case, the generation is obtained due to amplification of transverse electromagnetic waves (as in quantum cascade lasers, hot-carrier $p$-Ge lasers, 
etc.) which exploit population inversion between some energy states. In each of these ways, the generation efficiency decreases significantly when trying to extend the generation into the $\mathrm{THz}$ region. Therefore, the individuation of physical mechanisms which can lead to $\mathrm{THz}$ generation making use of both the ways is a mandatory issue. The aim of this communication is to show that, due to the dynamic character of the associated NDC, the electron transit-time resonance (TTR) in momentum and real spaces under low-temperature optical-phonon emission is a promising mechanism to address such an issue. To support this conjecture we shall review recent results on $\mathrm{THz}$ generation due to optical-phonon emission assisted TTR in III-V and nitride-based 3D bulk materials, 2D quantum heterostructures, and submicron or overmicron $n^{+} n n^{+}$diodes.

\section{Transit-time resonance}

At low lattice temperature the momentum space is subdivided into two regions with respect to the optical phonon emission: the so-called passive and active regions characterized, respectively, by long and short scattering times, $\tau_{-}$and $\tau_{+}$, determined by relatively slow impurity and acoustic scatterings in the former region and fast optical phonon emission in the latter one (see Fig. 1a). As a
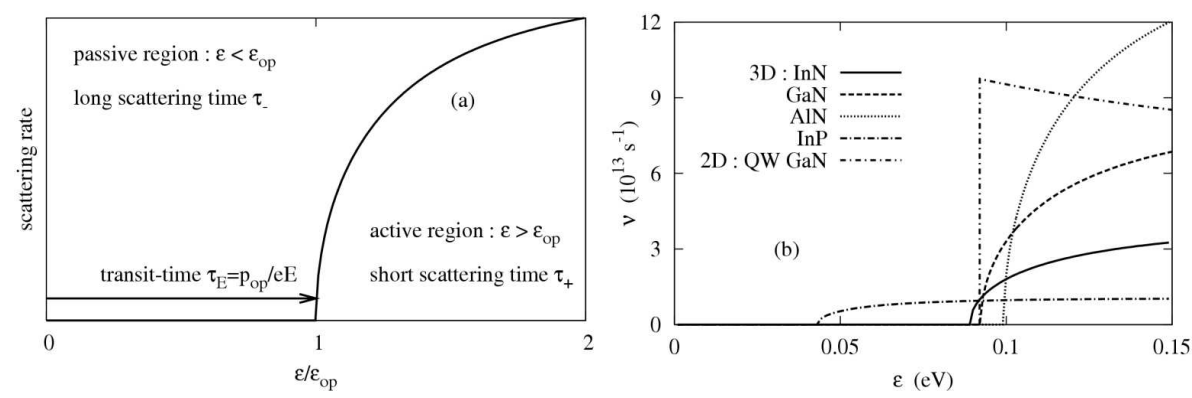

Fig. 1. (a) Schematic representation of the momentum space subdivision into the passive and active regions with respect to optical-phonon emission. (b) Scattering rate of polar-optical-phonon emission in 3D bulk materials and 2D GaN $5 \mathrm{~nm}$ QW.

result, because of acceleration from applied electric field, the carrier moves quasiballistically up to passive area boundary, then quickly emits an optical phonon and returns back into the passive region center where it starts the next cycle of acceleration thus providing a cyclic motion in the momentum space.

An increase in both the scattering intensity in the passive region and the electron penetration into the active region destroys the cyclic motion. This is usually formulated in terms of lower- and upper-limits for the transit time value $\tau_{E}=p_{0} / e E$ required for electron to cross ballistically the optical phonon hemisphere with radius $p_{0}$ in the momentum space under the applied electric field $E$ : 


$$
\tau_{-} \gg \tau_{E} \gg \tau_{+} .
$$

To fulfill the left inequality reasonably pure materials and low lattice temperatures are necessary. To satisfy the right inequality, the materials with strong interaction with optical phonon are preferable. In bulk 3D semiconductors this is achieved in nitrides as illustrated by Fig. 1b. Additional possibilities are provided by $2 \mathrm{D}$ quantum wells where the step-like onset of optical phonon emission (see Fig. 1b) leads to a further decrease in $\tau_{+}$.

The cyclic motion of carriers in the momentum space must manifest itself also in the real space at the scale of free-flight length necessary for an electron to reach the optical phonon energy $\hbar \omega_{0}: l_{0}=\hbar \omega_{0} / e E$. By comparing this quantity with the characteristic length $d$ of a sample one can separate three main possibilities for microwave power generation.

(i) Bulk materials $\left(d \gg l_{0}\right)$. In this case any effects of carrier redistribution in real space along the applied field direction are of no importance and the carrier motion is governed by some homogeneous electric field. As was shown by analytical considerations [1] under conditions of pronounced cyclic dynamics of electrons in momentum space the real part of the small-signal mobility takes negative values near the TTR frequency $f_{E}=1 / \tau_{E}$ (the so called dynamic negative differential mobility (DNDM)). Such a situation corresponds to amplification and generation of transverse electromagnetic waves which propagate in a direction transverse to the applied electric field. To realize such a generation one needs a photonic-way where the generation is obtained by using laser-like approaches.

(ii) Submicron $n^{+} n n^{+}$diodes $\left(d \approx l_{0}\right)$ with single phonon emission crossing the structure. In this case spatial variations of electron concentration are selfconsistently coupled with the local electric field through the Poisson equation. As shown by Ryzhij et al. [2] such a self-consistent variation of concentration and field leads to microwave power generation due to plasma waves. Thus, such a situation corresponds to high-frequency electronic-way where longitudinal current instabilities are used to obtain the generation.

(iii) Overmicron $n^{+} n n^{+}$structures (as some intermediate case) with multiple emissions of optical phonons (typically of about $\left.d \approx(10-20) l_{0}\right)$. In this case the formation of a spatial modulation of electron concentration and accompanied quantities with period $l_{0}$ can take place (the so-called dynamic free-carrier grating [3]). Again, under certain conditions this situation is favorable for the $\mathrm{THz}$ radiation generation.

The common feature of all these cases is that the spectral behavior of the generation has a resonant character with respect to the transit-time frequency. Therefore, one can consider them as manifestation of the electron TTR assisted by the optical phonon emission process.

\section{Generation of transverse electromagnetic waves}

For amplification and generation of transverse electromagnetic waves to be possible the real part of the differential mobility must be negative in desirable fre- 

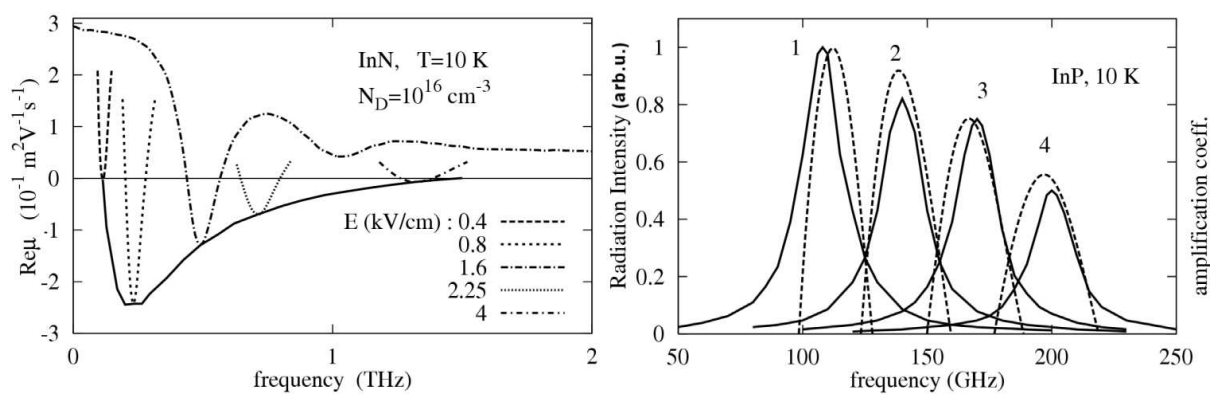

Fig. 2. (a) General behavior of the spectrum of the differential mobility real part (curve at $E=1.6 \mathrm{kV} / \mathrm{cm}$ ) and variation of the resonance region with electric field. Solid line refers to the maximum value of the DNDM as a function of frequency. (b) Intensity of microwave radiation measured experimentally [4] in InP samples at $E=250,300,350$ and $400 \mathrm{~V} / \mathrm{cm}$ (solid lines) and amplification coefficient calculated for bulk InP (dashed lines) at $T_{0}=10 \mathrm{~K}, E=217,267,317$ and $367 \mathrm{~V} / \mathrm{cm}$ (curves 1 to 4 , respectively) as function of frequency of the radiation.

quency range. The DNDM caused by optical phonon emission assisted transit-time resonance of electrons (shortly OPTTR) in bulk 3D materials and 2D quantum well (QW) and heterolayer (HL) structures entirely satisfies this condition. As an example, Fig. 2a presents the general shape of the differential mobility spectrum under OPTTR conditions (curve for $E=1.6 \mathrm{kV} / \mathrm{cm}$ ) and variation of its resonance region with electric field calculated by Monte Carlo (MC) procedure for InN at $10 \mathrm{~K}$. Solid line shows the maximum value of the DNDM. The appearance of a pronounced minimum of $\operatorname{Re}(\mu(f))$, which quickly reaches the negative values with increase in $E$ (curves for $E=0.4$ and $0.8 \mathrm{kV} / \mathrm{cm}$ ), is connected with the onset of a carrier periodic motion due to electron runaway from the low-energy region dominated mainly by impurity scattering which deteriorates the quasi-ballistic motion inside the optical phonon sphere. With a further increase in $E$ the maximum value of the DNDM decreases and finally goes to zero due to a significant penetration of carriers into the active region, which destroys the coherence of successive free flights. Thus, the electron runaway from intensive impurity scattering and the penetration into the active region are responsible for the low- and high-frequency (HF) cut-off of the generation in bulk semiconductors.

The microwave power generation related with the OPTTR was experimentally observed for the first time in $\mathrm{InP}$ [4]. Figure $2 \mathrm{~b}$ compares the spectra of generated microwave radiation intensity $I(f)$ experimentally measured in Ref. [4] in InP samples (solid lines) and the differential mobility real part, $-\operatorname{Re}(\mu(f))$, in the generation band calculated by MC method for bulk InP at $10 \mathrm{~K}$ and $N_{\mathrm{D}}=10^{15} \mathrm{~cm}^{-3}$ (dashed lines). The experimental sample was an n-InP rectangular parallelepiped with dimensions $54 \times 8 \times 7 \mathrm{~mm}$. Ohmic contacts were placed at $54 \times 7 \mathrm{~mm}$ edges. Mirrors were placed at $8 \times 7 \mathrm{~mm}$ edges. One of the mirror was slightly smaller 
than $8 \times 7 \mathrm{~mm}$ to provide the output of the generated radiation. Thus the geometry of the experiment corresponds to amplification and generation of transverse electromagnetic waves. Both experimental and theoretical curves are normalized to the maximum values of, respectively, $I$ and $-\operatorname{Re}(\mu)$ for the curve 1 . A good qualitative and reasonable quantitative agreement is evident.

Figure 3a reports and compares results of MC simulations of the OPTTR generation frequency band as function of the applied dc field for various $3 \mathrm{D}$ and $2 \mathrm{D}$ semiconductor structures. The low-frequency cut-off of the generation is related with the violation of quasi-ballistic motion of electrons in the passive region (left inequality in Eq. (1)), while the HF cut-off is related with the increasing penetration of electrons into the active region (right inequality in Eq. (1)). Quantitative estimations of these qualitative constraints obtained on the basis of numerical calculations of the OPTTR DNDM by the Monte Carlo method in various 3D materials and 2D QWs show that [5]: (i) for the left inequality the average momentum relaxation time in the passive region must satisfy the condition $\tau_{-} \geq(1 \div 2) \tau_{E}$, (ii) for the right inequality carrier penetration into the active region, $\delta \varepsilon / \hbar \omega_{0}$ must occur at a level smaller than $14 \div 15 \%$.
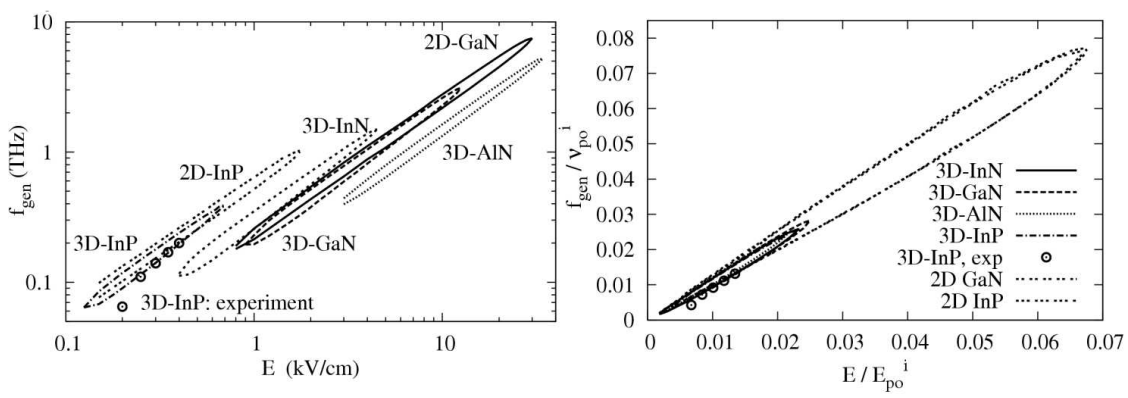

Fig. 3. (a) Generation bands (frequency regions where the real part of the differential mobility is negative) as function of applied dc field calculated by MC method for bulk materials as well as $12 \mathrm{~nm} \mathrm{InP}$ and $5 \mathrm{~nm}$ GaN QW structures at $10 \mathrm{~K}$. Points show the generation frequency obtained experimentally by Vorob'ev et al. [4]. (b) The same dependences normalized to characteristic po-scattering fields $E_{\mathrm{po}}^{i}$ and frequency $\nu_{\mathrm{po}}^{i}[5]$.

As shown in [5], by using an analytical model based on phonon scattering rates and ideal streaming motion these limit values allow one to estimate the lower- and upper-frequency limits of the generation band. Having in mind that for $\mathrm{THz}$ radiation generation the $\mathrm{HF}$ limit is of most interest, for the $\mathrm{HF}$ cutoff of the DNDM it was found: (i) for 3D bulk materials the relevant physical quantities to describe the HF cut-off are the carrier effective mass, the optical phonon energy, and the polar-optical coupling strength; (ii) in passing from 3D to $2 \mathrm{D}$ lateral transport, for the same material the influence of $2 \mathrm{D}$ transport on the OPTTR is characterized entirely by the dimensionless parameter $k_{0} d$ related 
with both the radius of the optical phonon sphere in wave vector space $k_{0}$ and the effective width of the electron localization $d$ associated with the lowest miniband of the QW/HL structure; (iii) in going from 3D to 2D transport, the change of the energy dependence of the density-of-states is responsible for an extension of the maximum generation frequency for up to a factor of five times. Moreover, such a model provides the universal representation of the generation band associated with OPTTR by using the electric field $E$ and the generation frequency $f_{\text {gen }}$ normalized, respectively, to the effective electric field of polar-optical phonon emission $E_{\mathrm{po}}^{i}$ and the effective scattering rate $\nu_{\mathrm{po}}^{i}=e E_{\mathrm{po}}^{i} / p_{0}(i=3 \mathrm{D}, 2 \mathrm{D})$, as shown in Fig. 3b.

\section{Longitudinal current instabilities in $n^{+} n n^{+}$diodes}

The current-voltage relation (CVR) for submicron $\mathrm{InN}$ diode is shown in Fig. 4a. The enhanced current spread of CVR is due to nearly-harmonic current oscillations appearing in instability areas at $u_{0}<U<2 u_{0}$ and $2 u_{0}<U<3 u_{0}$ (with $U$ being the applied voltage and $u_{0}=\hbar \omega_{0} / e$ ) corresponding, respectively, to one and two optical phonon emission when an electron crosses the diode. The
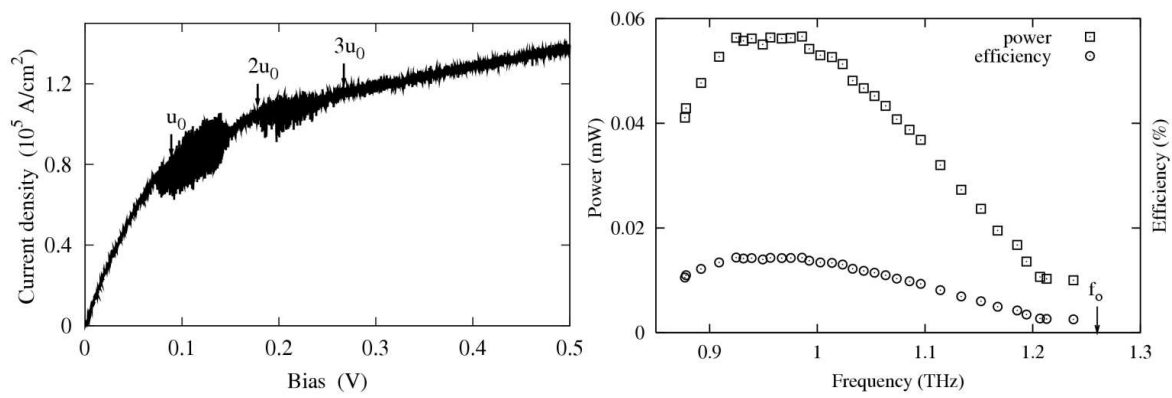

Fig. 4. (a) Current-voltage relation of $0.02-0.05-0.3-0.02 \mu m n^{+} n^{-} n n^{+}$InN diode with dopings $n^{-}=5 \times 10^{15}, n=3 \times 10^{16}, n^{+}=10^{18} \mathrm{~cm}^{-3}$ calculated by MC method at $80 \mathrm{~K}$ under voltage driven operation, and (b) the generated power for the same diode operating in a parallel resonant circuit.

generated power and efficiency calculated for the diode operation in the parallel resonant circuit is shown in Fig. 4b. Thus, in this case the diode can generate the $\mathrm{THz}$ radiation both with and without external resonant circuit. The formation of a dynamical spatial free-carrier grating in long $n^{+} n n^{+}$structures is illustrated in Fig. 5a. A low energy electron injected from the emitter into the $n$-region with energy $\varepsilon \ll \hbar \omega_{0}$ is accelerated by the field up to the optical phonon energy and then it loses energy and velocity after optical phonon emission. This process is repeated until the electron leaves the $n$-region through the collector. As a consequence, the electron trajectory consists of a sequence of free flight and stop events. Therefore, we observe the formation of a free-carrier grating with period $l_{0}=\hbar \omega_{0} / e \bar{E}$, where $\bar{E} \approx U / L$ is the average field in the $n$-region. The electron transport within one 

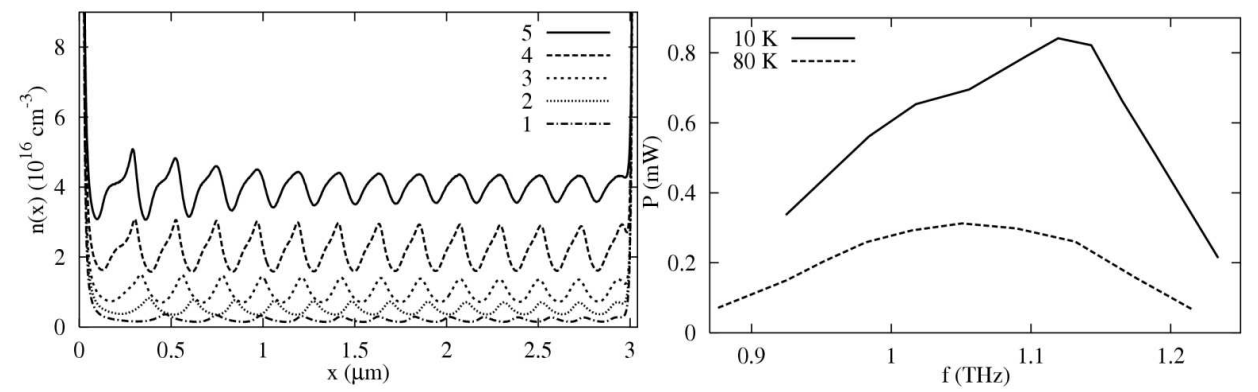

Fig. 5. (a) Concentration profiles in the $n$-region of an $n^{+} n n^{+} \mathrm{InN}$ structure at $80 \mathrm{~K}$, $1.2 \mathrm{~V}$ bias and different dopings $n$ of the $n$-region: curves from 1 to 5 correspond to $n=0.2,0.5,1,2.2$, and $4 \times 10^{16} \mathrm{~cm}^{-3}$ and (b) generated power spectra of an InN diode with $N=2.2 \times 10^{16} \mathrm{~cm}^{-3}$ operating in a parallel resonant circuit at 10 and $80 \mathrm{~K}$.

grating period can be considered as a transport in a sequence of short $n^{+} n n^{+}$ substructures with length $l_{0}$, since a stop event serves as the emitter and collector for two neighboring substructures at the same time. The frequency dependence of the $\mathrm{THz}$ power generated by $\mathrm{InN}$ diode of Fig. 5a connected in parallel with the resonant circuit is reported in Fig. 5b. By comparing Fig. 5b with Fig. 4b a considerable increase in the generated power is evident. This is due to the fact that a long diode can be treated as a net of shorter subdiodes connected in series.

\section{Noise as indicator of DNDM and current instabilities}

Here we stress that the resonant enhancement of the velocity and/or current noise under OPTTR conditions can be considered as an indicator of pronounced cyclic dynamics and can help to estimate experimental conditions necessary for the generation. This is illustrated by Fig. 6 which presents velocity (Fig. 6a) and current noise (Fig. 6b) in bulk $\mathrm{InN}$ and an $n^{+} n n^{+} \mathrm{InP}$ diode, respectively.
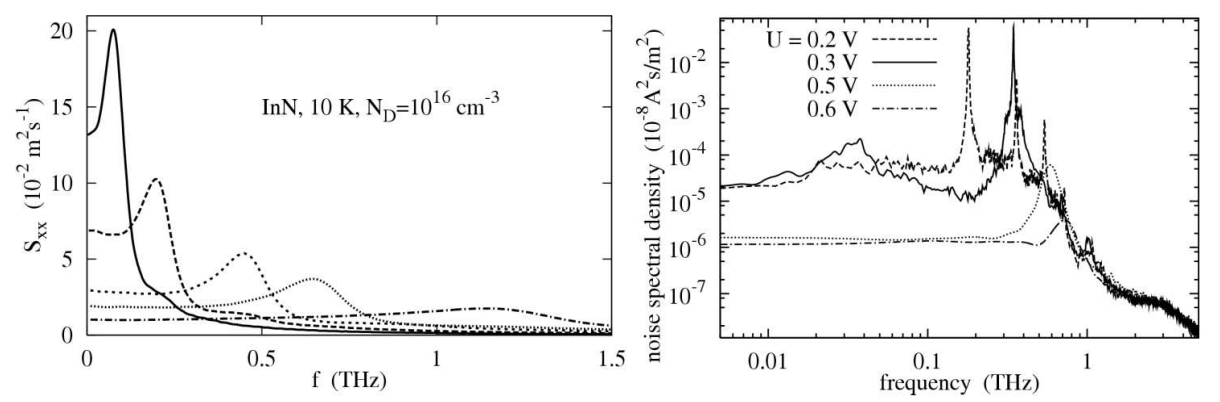

Fig. 6. (a) Spectral density of velocity fluctuations calculated by MC method for bulk InN at the same conditions of Fig. 2a, and (b) spectral density of current fluctuations of 0.02-5-0.02 $\mu m n^{+} n n^{+} \operatorname{InP}$ diode with $n=10^{15}$ and $n^{+}=10^{17} \mathrm{~cm}^{-3}$. 


\section{Acknowledgments}

This work was supported, in part, by French-Lithuanian project Gilibert and Lithuanian State Science and Studies Fundation contract No P-01/2007.

\section{References}

[1] A.A. Andronov, V.A. Kozlov, JETP Lett. 17, 87 (1973).

[2] V.I. Ryzhij, N.A. Bannov, V.A. Fedirko, Fiz. Tekh. Poluprovodn. 18, 769 (1984).

[3] V. Gružinskis, E. Starikov, P. Shiktorov, Acta Phys. Pol. A 107, 163 (2005).

[4] L.E. Vorob'ev, S.N. Danilov, V.N. Tulupenko, D.A. Firsov, JETP Lett. 73, 219 (2001).

[5] P. Shiktorov, E. Starikov, V. Gružinskis, L. Varani, C. Palermo, J.-F. Millithaler, L. Reggiani, Phys. Rev. B 76, 045333 (2007). 